\title{
WEAKLY COMPACT HOMOMORPHISMS FROM C*-ALGEBRAS ARE OF FINITE RANK
}

\author{
MARTIN MATHIEU
}

(Communicated by Paul S. Muhly)

\begin{abstract}
We give a straightforward proof of the fact that every weakly compact homomorphism from a $C^{*}$-algebra is a finite rank operator.
\end{abstract}

In [3] Ghahramani proved that every compact homomorphism between $\mathrm{C}^{*}$ algebras is a finite rank operator. Recently, Galé and Ransford [2] obtained the same conclusion under the more general hypothesis of weak compactness by first proving the result for the Wiener algebra and then applying Newburgh's theorem on the continuity of the spectral radius as well as Aupetit's scarcity theorem. The purpose of this note is to present a very simple proof that merely uses some basic facts of functional analysis.

Theorem. Every weakly compact homomorphism from a $C^{*}$-algebra into a normed algebra is of finite rank.

Proof. Let $\pi: A \rightarrow B$ be such a homomorphism. Then, $\tilde{A}=A / \operatorname{ker} \pi$ is a $C^{*}$ algebra and we have a monomorphism $\tilde{\pi}: \tilde{A} \rightarrow B$ defined by $\tilde{\pi} \circ \rho=\pi$, where $\rho$ is the canonical quotient map. Since $\rho$ is open, every bounded subset of $\tilde{A}$ is the image of a bounded set in $A$; thus $\tilde{\pi}$ is weakly compact. Let $x \in A$. By Gelfand theory, the restriction of $\tilde{\pi}$ to the abelian $\mathrm{C}^{*}$-subalgebra generated by $\rho\left(x^{*} x\right)$ is spectral radius preserving. Since the spectral radius coincides with the norm for positive elements, we conclude that

$$
\|\rho(x)\|^{2}=\left\|\rho(x)^{*} \rho(x)\right\| \leq\left\|\tilde{\pi}\left(\rho(x)^{*} \rho(x)\right)\right\| \leq\|\tilde{\pi}\|\|\rho(x)\|\|\tilde{\pi}(\rho(x))\| .
$$

Thus, $\tilde{\pi}$ is bounded below. Denoting by $\tilde{\pi}^{-1}: \tilde{\pi}(\tilde{A}) \rightarrow \tilde{A}$ a continuous inverse of $\tilde{\pi}$, we infer that $\mathrm{id}_{\tilde{A}}=\tilde{\pi}^{-1} \circ \tilde{\pi}$ is weakly compact whence $\tilde{A}$ is reflexive. But a reflexive $C^{*}$-algebra is finite dimensional which yields that $\pi(A)=\tilde{\pi}(\tilde{A}) \cong$ $A / \operatorname{ker} \pi$ is finite dimensional. This completes the proof.

Received by the editors February 23, 1989 and, in revised form, May 22, 1989. This paper was written while the author was a Visiting Fellow at the Centre for Mathematical Analysis of the Australian National University in Canberra, and was presented at the Great Plains Operator Theory Seminar held in Houston, Texas on May 4, 1989. The support received from the CMA, the DFG, and the NSF is gratefully acknowledged.

1980 Mathematics Subject Classification (1985 Revision). Primary 46 L 05, 47 B 99.

Key words and phrases. Weakly compact homomorphisms, $\mathrm{C}^{*}$-algebras. 
Remark. The fact that every continuous isomorphism from a $\mathrm{C}^{*}$-algebra is bounded below seems to have been noticed first in [1, Lemma 5.3].

\section{REFERENCES}

1. S. B. Cleveland, Homomorphisms of non-commutative *-algebras, Pacific J. Math. 13 (1963), 1097-1109.

2. J. Galé, T. J. Ransford, Weakly compact homomorphisms of Banach algebras, preprint.

3. F. Ghahramani, Compact homomorphisms of $C^{*}$-algebras, Proc. Amer. Math. Soc. 103 (1988), 458-462.

Mathematisches Institut, Universität Tübingen, Auf Der Morgenstelle 10, D-7400 Tübingen, Federal Republic OF Germany 